Article

\title{
Reversible Low-Light Induced Photoswitching of Crowned Spiropyran-DO3A Complexed with Gadolinium(III) Ions
}

\author{
Klaus Kruttwig ${ }^{1}$, Diego R. Yankelevich ${ }^{2}$, Chantal Brueggemann ${ }^{3}$, Chuqiao Tu ${ }^{1}$, \\ Noelle L'Etoile $^{3}$, André Knoesen ${ }^{2}$ and Angelique Y. Louie ${ }^{1, *}$ \\ 1 Department of Biomedical Engineering, University of California, Davis, CA 95616, USA \\ 2 Department of Electrical and Computer Engineering, University of California, Davis, CA 95616, \\ USA \\ 3 Department of Cell and Tissue Biology, University of California, San Francisco, CA 94143, USA \\ * Author to whom correspondence should be addressed; E-Mail: aylouie@ucdavis.edu; \\ Tel.: +1-530-752-7134; Fax: +1-530-752-5739.
}

Received: 26 March 2012; in revised form: 11 May 2012 / Accepted: 25 May 2012 /

Published: 31 May 2012

\begin{abstract}
Photoswitchable spiropyran has been conjugated to the crowned ring system DO3A, which improves its solubility in dipolar and polar media and stabilizes the merocyanine isomer. Adding the lanthanide ion gadolinium(III) to the macrocyclic ring system leads to a photoresponsive magnetic resonance imaging contrast agent that displays an increased spin-lattice relaxation time $\left(T_{1}\right)$ upon visible light stimulation. In this work, the photoresponse of this photochromic molecule to weak light illumination using blue and green light emitting diodes was investigated, simulating the emission spectra from bioluminescent enzymes. Photon emission rate of the light emitting diodes was changed, from $1.75 \times 10^{16}$ photons $\cdot \mathrm{s}^{-1}$ to $2.37 \times 10^{12}$ photons $\cdot \mathrm{s}^{-1}$. We observed a consistent visible light-induced isomerization of the merocyanine to the spiropyran form with photon fluxes as low as $2.37 \times 10^{12}$ photons $\cdot \mathrm{s}^{-1}$ resulting in a relaxivity change of the compound. This demonstrates the potential for use of the described imaging probes in low light level applications such as sensing bioluminescence enzyme activity. The isomerization behavior of gadolinium(III)-ion complexed and non-complexed spiropyran-DO3A was analyzed in water and ethanol solution in response to low light illumination and compared to the emitted photon emission rate from over-expressed Gaussia princeps luciferase.
\end{abstract}


Keywords: spiropyran; photochromic molecule; DO3A; MRI contrast agent; luciferase; light emitting diodes; rate constant; low light applications

\section{Introduction}

Spiropyrans represent the most widely studied class of organic photochromes, but the exact mechanism of photocoloration has yet to be determined [1]. In general, spiropyran (SP) is stable in its closed-ring isomeric form, and is a colorless or pale yellow solution in non-polar solvents. After exposure to UV irradiation, this SP form is converted to a metastable open-ring isomer (merocyanine, $\mathrm{MC}$ ), possessing an optical absorption peak at 550-600 $\mathrm{nm}$. The original colorless SP form, can be restored via visible light irradiation and/or thermal induction. Conjugation of a nitrogroup into the benzene ring of the chromene part of spiropyran acts as a $\pi$-accepting substituent [2]. This leads to a strong bathochromic shift in the open-chain isomer [1]. Spiropyrans in their closed form are soluble in a wide range of organic solvents and display quite low water solubility [3]. This impairs/restricts their use in cellular biological applications, where water solubility is required. In fact, spiropyran isomerization and effects of substitutions on the isomerization have been well investigated in organic solvents, whereas little is known about those in aqueous solution [4].

Water-soluble spiropyrans have been synthesized by the introduction of a sulfonate group on the phenyl ring or 8-methoxy-6-nitro-BIPS [5]. The conjugation of a crown ether moiety is considered necessary to stabilize the merocyanine form through metal ion complexion formation and to produce a polar environment, inducing similar properties as those found with the application of polar solvents [6]. Substitution of spiropyrans with monoaza-15-crown-5, 15-crown-5 fragments or acyclic analogs in position 6' or the nitrogen atom of the indoline ring, as well as in position 8' of the benzopyrene part has a large influence on the photochromic properties of the molecule [7]. It has been reported that a crowned metal cation is capable of forming a coordination bond with the phenolate oxygen anion, which eventually leads to the stabilization of the merocyanine form. Photoirradiation of crown-containing spiropyrans leads to a transformation into the closed form of the molecule with a subsequent release of the metal ion.

Previously we have reported the conjugation of spiropyran to a DO3A-macrocycle and further complexion of a gadolinium ion $\left(\mathrm{Gd}^{3+}\right.$ ion) and successful application as light responsive Magnetic Resonance Imaging (MRI) contrast agent (Scheme 1) [2,8]. Due to the electrostatic interaction of the complexed metal ion with the indoline part of the photochromic molecule, the hydration state of $\mathrm{Gd}(\mathrm{III})$ is altered depending on the isomerization state of spiropyran, resulting in a modification of the contrast agent relaxivity. Photochromism of spiropyrans is affected by several factors, such as the temperature, the nature of the solvent and the substitution of side groups. The light power density (irradiance) that has been reported required to initiate a photochromic conversion ranges from below $0.9 \mathrm{~mW} \cdot \mathrm{cm}^{-2}$ up to several $100 \mathrm{~mW} \cdot \mathrm{cm}^{-2}$, but most of the reports in the literature are lacking precise photometric details [9-16]. 
Scheme 1. Structural representation of spiropyran-DO3A-Gd(III): Proposed isomerization of the compound, displaying the two conformational states: merocyanine (left) and spiropyran (right). Under dark conditions, spiropyran-DO3A is preferentially in the merocyanine $(\mathrm{MC})$ form, whereas visible light illumination leads to isomerization to the spiropyran form (SP).
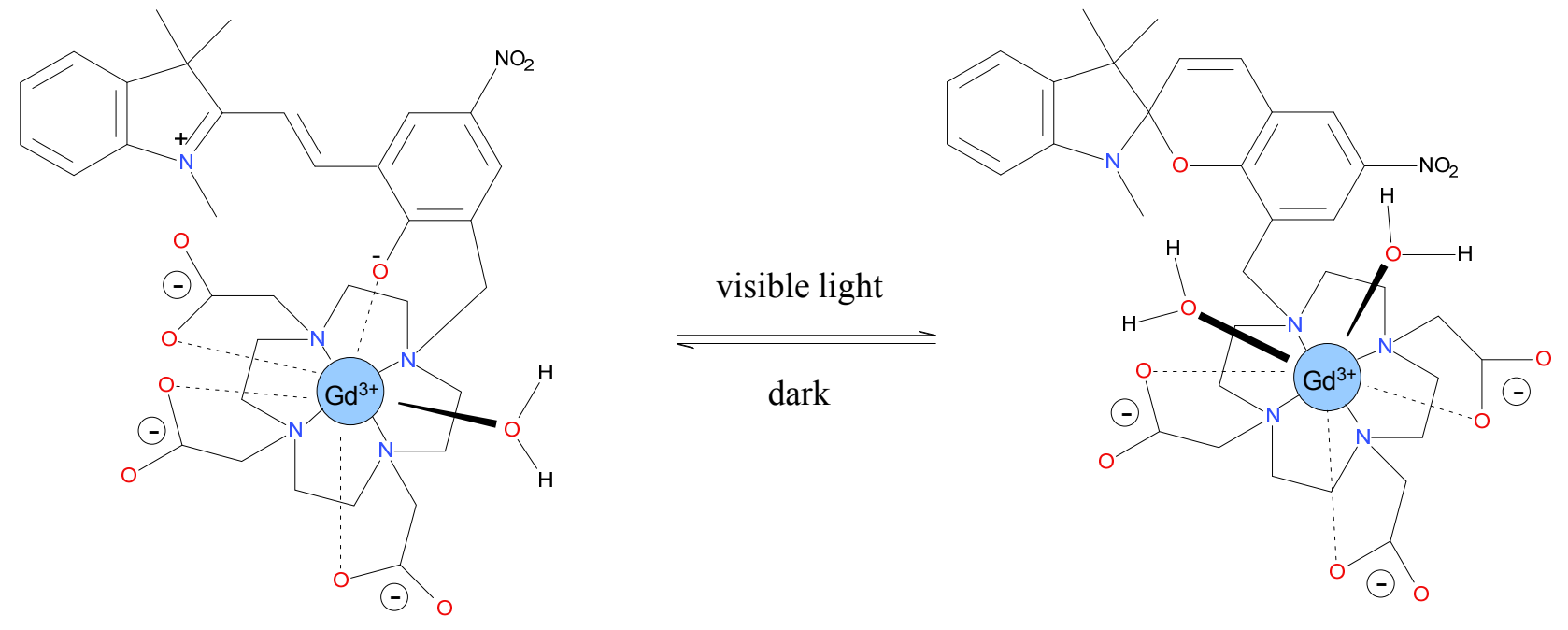

Recently, studies have demonstrated that low power irradiation using light emitting diodes (LEDs, with an irradiance of approximately $1 \mathrm{~mW} \cdot \mathrm{cm}^{-2}$ ) can be used to effectively trigger the SP to MC isomerization $[9,17]$. In fact, Yoshida et al. demonstrated a linear correlation between switching time and laser power in spiropyran-doped film [18]. They reported a maximum response time of 200-300 ms with a power of approx. $50 \mathrm{~mW}$ of the laser at $\lambda=532 \mathrm{~nm}$. But, the rate constants and irradiance associated with these results were not reported [18]. To our knowledge, there have been no systematic studies reported that analyze the effect of low light illumination on spiropyran-based molecules possessing a photostable MC state. Measuring the amount of light capable of inducing photochromic conversions is of particular importance for experimental studies where light levels are restricted, such as in biological tissue applications. There, the visible light penetration depth is limited, dependent on the wavelength, the absorption, and scattering properties of the tissue [19,20]. This limited light penetration would seem to preclude the in vivo application of light switchable molecules, which have great potential as pharmaceutical and imaging systems. Examples of the use of spiropyrans in vivo have been reported, with some degree of success. For example, Ipe et al. described a spiropyran-based drug release system, where the amino acid derivative L-3,4-dihydroxyphenylalanine (L-DOPA) could be released upon light stimulation [21]. Additionally, an antibody-mediated targeting of a spiropyran containing a two-photon imaging probe to breast cancer cells in vitro has been recently described [22]. These are key developments for future in vivo studies for selective tumor cell labeling, light induced release of pharmaceutics, and the application of contrast agents for non-invasive imaging. These studies clearly demonstrate the emerging potential of reversible photochromic molecules in diagnostic and therapeutic applications.

Our group has previously described the synthesis and proof-of-principle application for a DO3A conjugated spiropyran and dinitrospiropyran to be used as a reversible MRI imaging probe in vivo [2,8]. Understanding both the photochromic behavior and the relaxivity properties (e.g., the spin-lattice 
relaxation time $T_{1}$, etc.) of spiropyran-based probes in response to light irradiation is necessary for advancing its biological applications. Herein, we describe our investigation of the switching behavior of spiropyran-DO3A using defined spectral irradiation properties, mimicking emission from a bioluminescent enzyme with low power illumination in different solvents. Emission of a bioluminescence signal refers to the generation of visible light from an enzyme-catalyzed reaction of molecular oxygen with the substrate luciferin [23]. Firefly luciferase (FLuc) from Photinus pyralis is one of the most commonly used luciferases in applications of bioluminescence, exhibiting a broad spectral emission with a peak at $562 \mathrm{~nm}$ [24,25]. The click beetle luciferase from Pyrearinus termitilluminans, displays a maximum light emission at $\lambda=538 \mathrm{~nm}$ and the highest quantum yield reported yet of 0.61 [26,27]. Alternatively, ATP-independent luciferases, using coelenterazine as a substrate like Gaussia princeps luciferase (GLuc) with a peak light emission at $470 \mathrm{~nm}$ can be used [28]. A codon-optimized variant with a 200 fold (in vivo) to 1000 fold (in vitro) higher bioluminescence signal intensity when compared to FLuc and Renilla luciferase (RLuc) will be used in our study to compare LED emitted photon flux with enzymatically produced light [29,30]. Overall, we describe a novel system in which spiropyran tethered MRI contrast agents respond to low light such that MRI may be used to map the expression of bioluminescent markers. In addition, we report the properties of previously developed set of novel light responsive MRI probes that can be reversibly activated by illumination with visible light $[2,8]$.

\section{Results and Discussion}

\subsection{Spectroscopic Characteristics of Gd(III) Complexed and Non-Complexed Spiropyran-DO3A}

Peak absorbance values for the crowned spiropyran and crowned spiropyran complexed with $\mathrm{Gd}$ (III) ions were adjusted to $0.5-0.6$ in water and 0.6-0.7 in EtOH, respectively. Spiropyran-DO3A dissolved in water displayed two peak absorption bands, one in the region between $\lambda=300 \mathrm{~nm}$ to $400 \mathrm{~nm}$ with a maximum at $350 \mathrm{~nm}$ and one from $400 \mathrm{~nm}$ to $550 \mathrm{~nm}$ displaying a peak absorbance of 0.514 $( \pm 0.011)$ at $496 \mathrm{~nm}$ (Figure 1A). A red shift and increase of the peak absorption peak to $0.606( \pm 0.02)$ at $520 \mathrm{~nm}$ was observed when the contrast agent was dissolved in ethanol solution (Figure 1A).

In contrast to spiropyrans bearing free hydroxy, carboxy or amino groups either on the indoline or the benzopyran part, which exhibit negative photochromism, the DO3A tethered spiropyran displayed positive photochromism in both the solvents water and ethanol [5]. Illumination for 1 min with the blue LED and a total photon emission rate of $1.754 \times 10^{16}$ photons $\cdot \mathrm{s}^{-1}$ resulted in a significant decrease of the absorbance values from $0.514( \pm 0.011)$ to $0.01\left( \pm 6.82 \times 10^{-4}\right)$ in the water solution, which represents an illumination-induced change of $98.05 \%$. A slightly larger absorbance decrease of $98.5 \%$ was detected after illumination ofspiropyran-DO3A in ethanol solution.

The Gd-complexed spiropyran-DO3A molecule displayed a peak absorption peak of $0.519( \pm 0.012)$ at $470 \mathrm{~nm}$ in water solution, which decreased to $0.165( \pm 0.012)$ after illumination (Figure 1B). The observed values are in accordance to the values reported previously by $\mathrm{Tu}$ et al. [8]. Dissolving spiropyran-DO3A-Gd in EtOH leads to a red-shifted peak of $0.547( \pm 0.019)$ at $478 \mathrm{~nm}$, which decreases to $0.066\left( \pm 4.28 \times 10^{-3}\right)$ after illumination (Figure 1B). A shift of the absorbance dependent on the surrounding environment, as observed in the current study, was described previously [31]. 
Interestingly, the observed hypsochromic wavelength shift, while increasing the polarity of the surrounding environment from $\mathrm{EtOH}$ to water, was three times larger when comparing non-complexed and Gd-complexed spiropyran-DO3A. The observed, photostationary stable merocyanine conformation has been described for crowned spirobenzopyrans earlier and may have its foundation in a strong electrostatic interaction between the high charge density of the phenoxide oxygen and the cyclen-complexed gadolinium cation [8].

Figure 1. Spectroscopic characteristics of spiropyran-DO3A complexed and non-complexed with Gd(III): Absorption of water and ethanol solutions of spiropyran-DO3A were spectroscopically analyzed (A). Illumination of the solutions leads to a decrease in absorption and a shift of the actinic band in the range between $350 \mathrm{~nm}$ and $400 \mathrm{~nm}$. A shift in the peak absorption wavelength of spiropyran-DO3A complexed with $\mathrm{Gd}(\mathrm{III})$ ions in ethanol and water could be detected (B). Illumination of spiropyran-DO3A-Gd in water leads to a decreased MC to SP conversion compared with the ethanol solution. Illumination was performed with a current of $18.5 \mathrm{~mA}$, corresponding to $1.754 \times 10^{16}$ photons $\cdot \mathrm{s}^{-1}$.

Spiropyran-DO3A

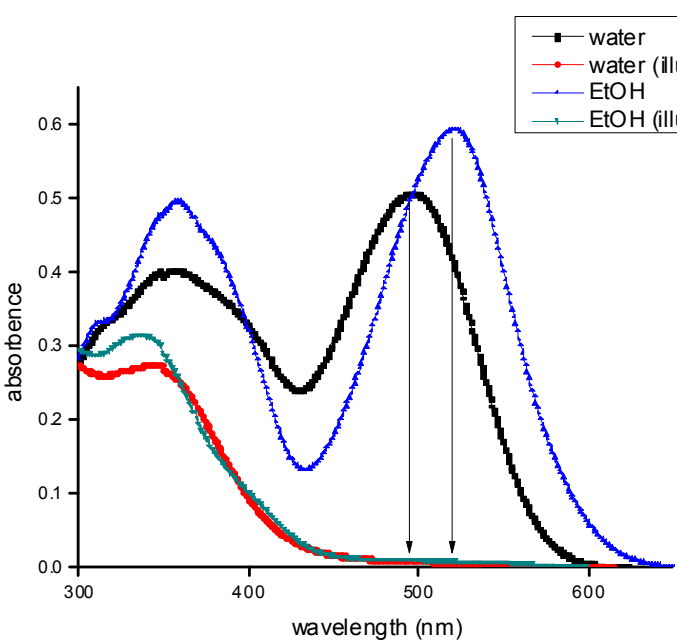

Spiropyran-DO3A-Gd

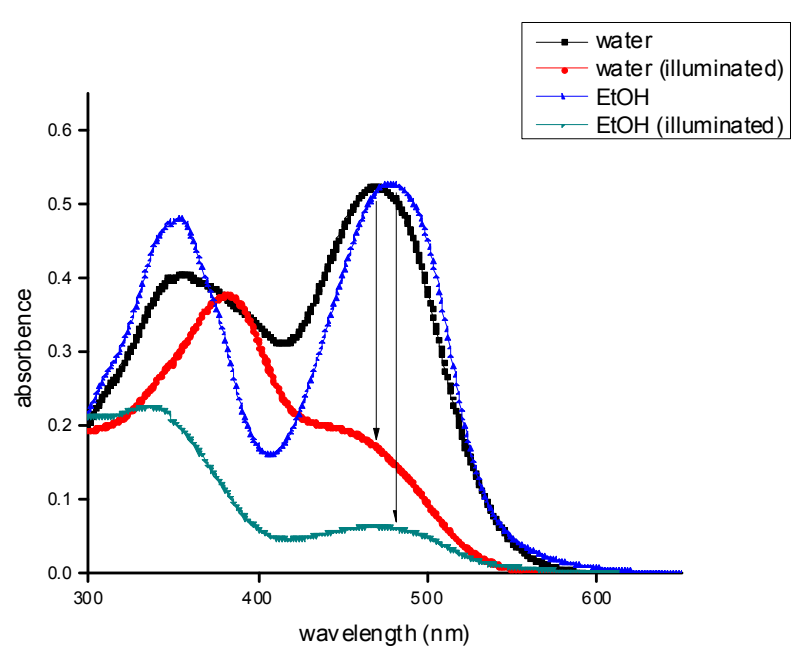

A

B

The length of the alkyl chain is one of the factors that influence the interaction between the metal ion and the photochromic part of the molecule [7]. Tethering of the DO3A macro complex to the photochromic molecule was achieved in the present investigation through a one carbon linker molecule (Scheme 1). The length of this linker has a pivotal role on switching efficiency, was previously shown by Diamond et al. using a covalently functionalized polystyrene bead-based system [17]. Varying the linker length from four to eight carbon atoms significantly improved the photochromic conversion of this polystyrene-bead-conjugated spiropyran [17].

\subsection{Correlation between Rate Constants and Total Photon Emission Rate}

Photokinetic analysis can be applied in the case of spiropyran-DO3A conjugated molecules, where thermal isomerization accompanies the photochemical processes and the rate of photostationary conversion depends on the irradiation wavelength and on the intensity of the incident photon flux [32]. 
For determination of rate constants for the light-induced MC to SP conversion of Gd(III), complexed and non-complexed spiropyran-DO3A, dissolved in water and/or ethanol, were illuminated for defined time periods. Spiropyran-DO3A was stable in the merocyanine form when dissolved in water and kept in the dark. It displayed absorbance fluctuations of approximately $5 \%$, but no gradual decrease of absorbance could be detected in contrast to spiropyran-DO3A dissolved in $\mathrm{EtOH}$. In EtOH, a decrease in absorbance values with a rate constant of $1.0 \times 10^{-3} \mathrm{~s}^{-1}$ was calculated under control (dark) conditions, clearly displaying a decreased half-life of the photostationary merocyanine state in EtOH as compared to water solution. Illumination of spiropyran-DO3A, dissolved in water with photon

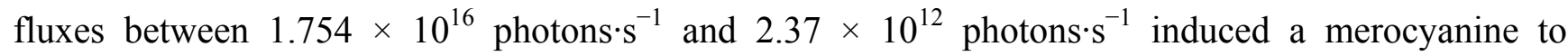
spiropyran isomerization. The light induced MC to SP isomerization process followed first order kinetics, as has been previously described [33]. The calculated rate constants were between $\mathrm{k}_{\mathrm{t}}=0.385$ $\left( \pm 3.4 \times 10^{-2}\right) \mathrm{s}^{-1}$ and $\mathrm{k}_{\mathrm{t}}=1.61 \times 10^{-4}\left( \pm 2.25 \times 10^{-5}\right) \mathrm{s}^{-1}$, displaying a linear regression coefficient of $\mathrm{R}^{2}=0.99156$ when dissolved in water and a linear regression coefficient of $\mathrm{R}^{2}=0.98982$ when dissolved in ethanol (Figure 2A). Illumination of spiropyran-DO3A dissolved in EtOH leads to significant higher rate constants as compared to the condition when the same molecule was dissolved in water. For the $\mathrm{MC}$ to $\mathrm{SP}$ isomerization of spiropyran-DO3A in ethanol under continuous illumination with $1.754 \times 10^{16}$ photons s ${ }^{-1}$, the rate constant was $\mathrm{k}_{\mathrm{t}}=0.653\left( \pm 4.5 \times 10^{-2}\right) \mathrm{s}^{-1}$. At the lowest photon count tested, irradiation with $1.50 \times 10^{13}$ photons $\cdot \mathrm{s}^{-1}$, a rate constant was $\mathrm{k}_{\mathrm{t}}=1.379 \times 10^{-3}$ $\left( \pm 1.56 \times 10^{-4}\right) \mathrm{s}^{-1}$. Because of the unstable MC state, we have not applied lower photon fluxes. It is noteworthy that spiropyran-DO3A possesses a maximal absorbance at $520 \mathrm{~nm}$, while the maximum emission of the blue LED is at $465 \mathrm{~nm}$. This implies that a higher rate constant can be achieved after ideal matching of the emission spectrum and the contrast agent absorbance spectrum, which we will be shown for the green LED in section 3.6.

The complexion of spiropyran-DO3A with $\mathrm{Gd}(\mathrm{III})$ ions leads to a superior stabilization of the merocyanine form. Under control (dark) conditions, fluctuations in absorbance values of $1.46 \%$ in water solution and $3.24 \%$ in ethanol solution have been observed. In contrast to spiropyran-DO3 dissolved in EtOH, no gradual decrease in absorbance could be detected under dark conditions. Illumination of spiropyran-DO3A-Gd dissolved in water with total photon rates between $1.754 \times 10^{16}$ photons $\cdot \mathrm{s}^{-1}$ and $2.37 \times 10^{12}$ photons $\cdot \mathrm{s}^{-1}$ induced a merocyanine to spiropyran isomerization with calculated rate constants between $\mathrm{k}_{\mathrm{t}}=0.3266( \pm 0.034) \mathrm{s}^{-1}$ and $\mathrm{k}_{\mathrm{t}}=1.28 \times 10^{-4}$ $\left( \pm 1.89 \times 10^{-5}\right) \mathrm{s}^{-1}$ and a linear regression coefficient of $\mathrm{R}^{2}=0.99978$ (Figure 2B). Illumination of spiropyran-DO3A-Gd, dissolved in EtOH does not lead to significantly higher rate constants compared to the water solution as observed for the non-complexed contrast agent. For the MC to SP isomerization under continuous illumination with $1.754 \times 10^{16}$ photons $\cdot \mathrm{s}^{-1}$, the rate constant was $\mathrm{k}_{\mathrm{t}}=0.358( \pm 0.049) \mathrm{s}^{-1}$. Illumination of the ethanol solution with $2.37 \times 10^{12}$ photons $\cdot \mathrm{s}^{-1}$ induced a merocyanine to spiropyran conversion with $\mathrm{k}_{\mathrm{t}}=1.21 \times 10^{-4}\left( \pm 1.21 \times 10^{-5}\right) \mathrm{s}^{-1}$. The linear regression coefficient was $\mathrm{R}^{2}=0.97866$. We were able to induce a MC to SP isomerization applying photon emission rates below the sensitivity range of the used optical power meter $\left(<2.37 \times 10^{12}\right.$ photons $\left.\cdot \mathrm{s}^{-1}\right)$ with spiropyran-DO3A-Gd dissolved in water, but not when dissolved in EtOH. While further decreasing the total photon emission rate with applying a current of $0.014 \mathrm{~mA}$ lead to a rate constant of $3.31 \times 10^{-5}\left( \pm 5.40 \times 10^{-6}\right) \mathrm{s}^{-1}$ in water, no MC to SP conversion was inducible in ethanol solution at this photon density. 
Figure 2. Determination of rate constants and maximum $\mathrm{MC}$ to $\mathrm{SP}$ conversion in relation to the total photon emission rate: The rate constants of spiropyran-DO3A-Gd, dissolved in water and ethanol were determined after illumination with the blue LED using equation (2). The insert displays the data points between $0 \mathrm{~s}^{-1}$ and $10 \times 10^{13} \mathrm{~s}^{-1}$. A linear regression analysis was performed which gave $\mathrm{R}^{2}=0.99156$ for spiropyran-DO3A dissolved in water and $\mathrm{R}^{2}=0.98982$ for spiropyran-DO3A dissolved in ethanol. For spiropyran-DO3A-Gd dissolved in water the regression analysis gave $R^{2}=0.99978$ and $R^{2}=0.97866$ for spiropyran-DO3A-Gd dissolved in EtOH.


To answer the question of what percentage of the spiropyran containing molecules can be efficiently interconverted to the spiropyran state at a defined photon emission rate, spiropyran-DO3A-Gd and spiropyran-DO3A in water or ethanol solution were illuminated until no further conversion was observed (Figure 3). Illumination of spiropyran-DO3A-Gd in water with a total photon emission rate of $1.754 \times 10^{16}$ photons $\cdot \mathrm{s}^{-1}$ leads to a change in absorbance of $62.46( \pm 2.44) \%$ whereas a isomerization of $86.95( \pm 4.71) \%$ could be induced in ethanol solution.

Decreasing the total photon rate leads to a decreased change in absorbance and a further extension of the illumination time needed for the interconversion. This impairment was significantly greater in ethanol solution compared with spiropyran-DO3A-Gd dissolved in water. Illumination with $2.37 \times 10^{12}$ photons $\cdot \mathrm{s}^{-1}$ lead to peak absorbance change of $50.86( \pm 3.56) \%$ when dissolved in water. This is in contrast to a peak absorbance change of $27.9( \pm 3.72) \%$ when dissolved in ethanol. At photon rates below $2.37 \times 10^{12}$ photons $\cdot \mathrm{s}^{-1}$ the peak absorbance changed markedly in water solution to $34.79( \pm 2.45) \%$, and no change was observed in ethanol solution (data not shown in Figure 3). To calculate the rate of the thermally-induced back-reaction after continuous illumination, we measured a 12-fold increase in the rate constant of spiropyran-DO3A when dissolved in ethanol versus water (Figure 3) at comparable absorbance values. 
Figure 3. Determination of maximum illumination-induced peak absorbance change in relation to the photon emission rate: The maximum peak absorbance change of spiropyranDO3A-Gd dissolved in water and ethanol for different photon emission rates intensities was determined after illumination with the blue LED. The total photon emission rate was calculated using Equation (3).

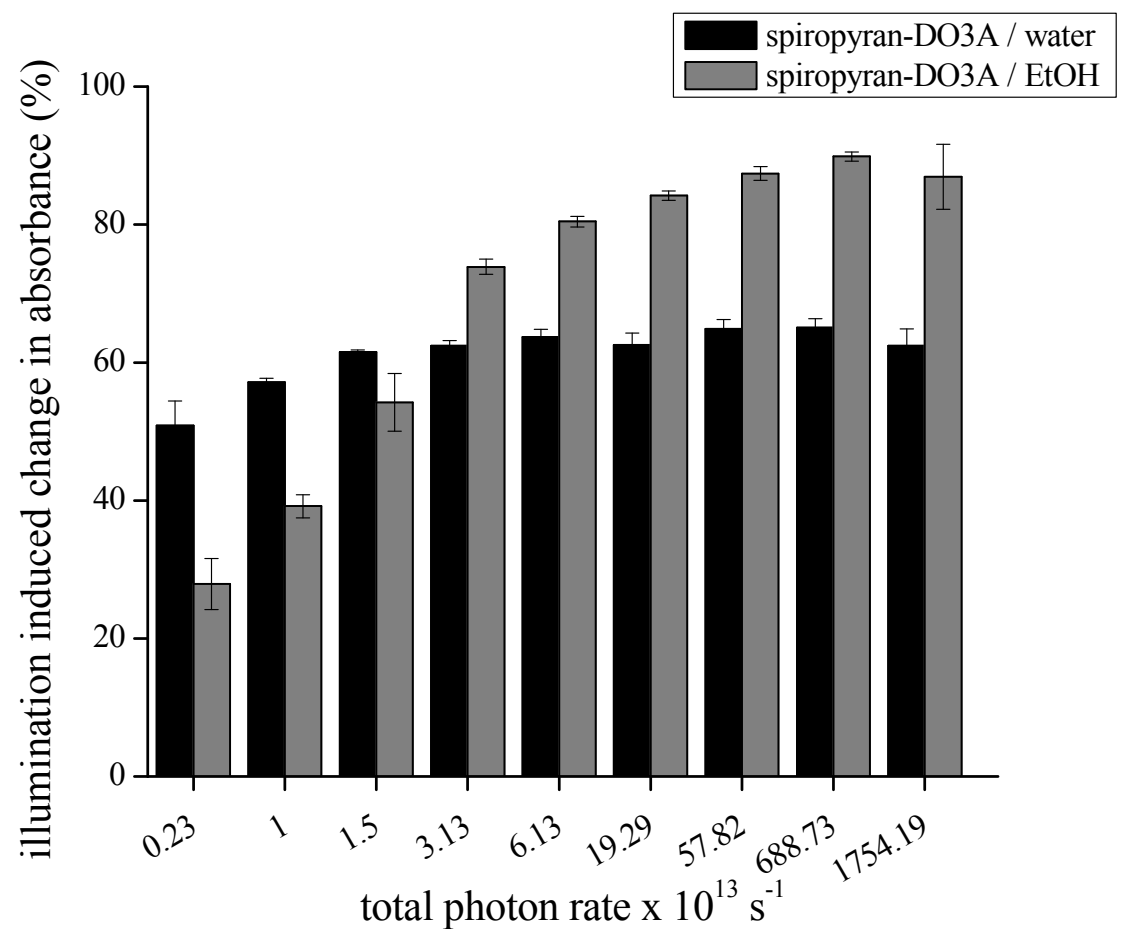

\subsection{Investigation of Increased Temperature on Back-Conversion and Repeated Switching Cycles}

Photodegradation of the merocyanine form of the spiropyran molecule is a well-known problem that limits the application of reversible, self-regenerating sensing molecules [34]. We focused further on describing the photochemical behavior of spiropyran-DO3A-Gd at physiologically relevant temperatures. Increasing the temperature from $25^{\circ} \mathrm{C}$ to $37{ }^{\circ} \mathrm{C}$ had no influence on the reaction kinetics observed while continuously illuminating spiropyran-DO3A-Gd dissolved in water. Also, the absorption spectra of the merocyanine form did not change at $37{ }^{\circ} \mathrm{C}$ during the course of the investigation, which is in accordance with the description from Movia et al. [35] In contrast to these observations while illuminating the sample, the thermally-induced back-reaction was affected by temperature. After terminating the irradiation for $1 \mathrm{~min}$ with a total photon emission rate of $1.754 \times 10^{16}$ photons $\cdot \mathrm{s}^{-1}$ the thermal relaxation rate was determined at $25{ }^{\circ} \mathrm{C}$ to be $\mathrm{k}_{\mathrm{t}}=1.1 \times 10^{-4}$ $\left( \pm 1.3 \times 10^{-4}\right) \mathrm{s}^{-1}$. This rate constant increased to $\mathrm{k}_{\mathrm{t}}=5.04 \times 10^{-4}\left( \pm 7.29 \times 10^{-4}\right) \mathrm{s}^{-1}$ at the temperature of $37^{\circ} \mathrm{C}$. We then investigated the influence of repeated switching cycles on the MC to SP conversion (Figure 4). When dissolved in water, switching of spiropyran-DO3A-Gd could be induced at least 3 times without impairment of complete restoration of the merocyanine form at $37{ }^{\circ} \mathrm{C}$. We focused on the thermodynamically induced interconversion to the merocyanine state instead of using UV illumination, which is known to induce photodegradation [17]. Also, for potential in vivo application of spiropyran-DO3A-Gd, it is critical to ensure the complete restoration of the merocyanine form without additional illumination. The time needed for complete restoration of the MC form was calculated to be 
approximately $174 \mathrm{~min}$. The rate constant for the thermal-induced back-reaction of spiropyran-DO3AGd in ethanol solution increased from $5.67 \times 10^{-3}\left( \pm 4.16 \times 10^{-3}\right) \mathrm{s}^{-1}$, determined at room temperature $1.52 \times 10^{-2}\left( \pm 1.20 \times 10^{-3}\right) \mathrm{s}^{-1}$ at $37^{\circ} \mathrm{C}$. The time needed for complete restoration of the MC form was calculated to be approximately $40 \mathrm{~min}$, when dissolved in EtOH.

Figure 4. Reversible interconversion between the spiropyran and the open merocyanine form: The thermal-induced back-reaction from the SP form to the MC form of spiropyranDO3A-Gd in water and ethanol solution was determined after illumination at a temperature of $37^{\circ} \mathrm{C}$. Blue arrows represent the irradiation time points of the water solution, while red arrows represent illumination of spiropyran-DO3A-Gd in ethanol.

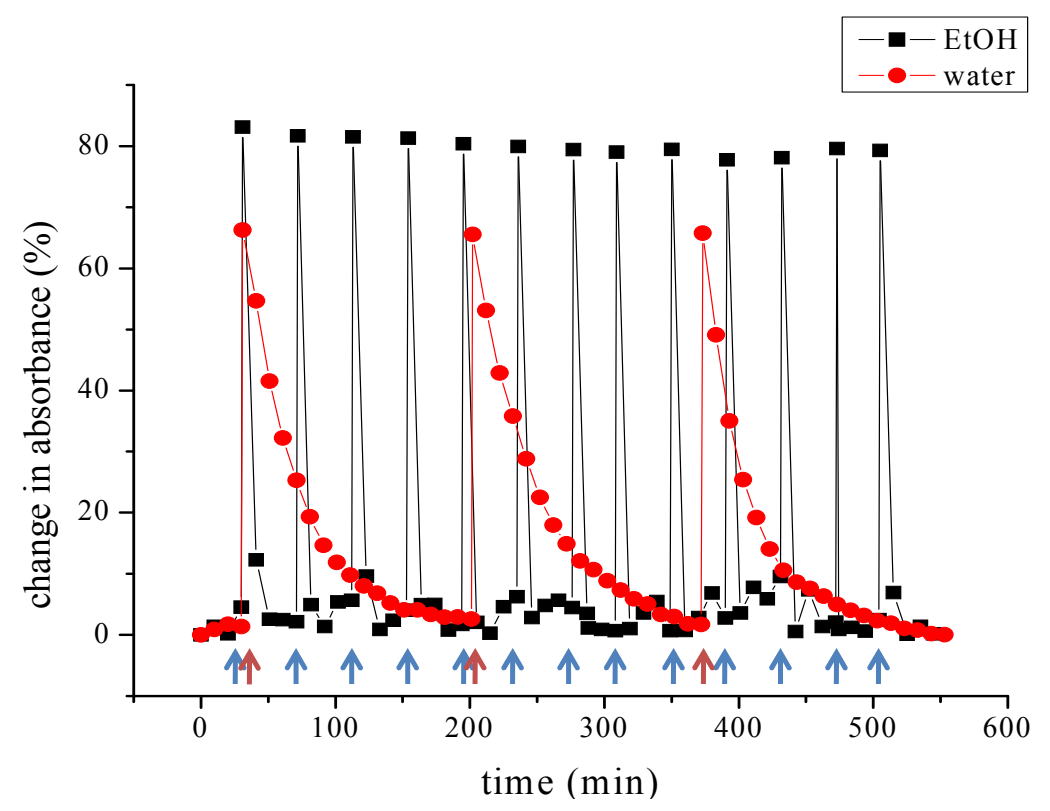

Our results, obtained with the application of several interconversion cycles between the spiropyran form and the merocyanine form, also clearly displayed that the amount of photobleaching is greatly reduced using low power LED illumination with $1.754 \times 10^{16}$ photons $\cdot \mathrm{s}^{-1}$ which has also been previously reported by Scarmagnani et al. [17].

\subsection{Relaxometric Properties}

Relaxometric properties of spiropyran-DO3A-Gd were analyzed using concentrations between $39.4 \mu \mathrm{M}$ and $157.6 \mu \mathrm{M}$. Spiropyran-DO3A-Gd kept under dark conditions possesses an $r_{1}$ of 2.93, whereas the $r_{1}$ value decreased to 2.63 after illumination for $60 \mathrm{~s}$ with the blue LED using a photon emission rate of $1.754 \times 10^{16}$ photons $\cdot \mathrm{s}^{-1}$. This represents a light-induced change in relaxivity of $10.24 \%$. The interaction of water molecules with the first coordination sphere of the Gd-containing contrast agents likely produces changes in the $T_{1}$ properties of the CA. The decrease in the $r_{1}$ relaxivity of the contrast agent upon visible light illumination may reflect the structural distinction between the merocyanine and spiropyran isomeric forms. In the spiropyran form, the complexed Gd(III) may be attracted to the indoline part of the photochromic molecule where electrostatic interactions between the metal ion and non-bonding electrons take place [2]. Thus, the indoline part influences water molecule access to the Gd(III) ion and impairs $T_{1}$ contrast generation. Experimentally, spiropyran-DO3A-Gd, dissolved in 
water was illuminated for different time periods with the blue LED, immediately followed by $T_{1}$ determination using relaxometry (Figure 5). The $\Delta T_{1}$ values obtained after applying different illumination intervals corresponds to the observed absorbance changes, determined with absorbance spectroscopy. The largest change in $T_{1}$ could be observed after 1 min irradiation with a total photon emission rate of $6.88 \times 10^{15}$ photons $\cdot \mathrm{s}^{-1}$.

Figure 5. Relaxometric properties of spiropyran-DO3A-Gd in water: Determination of the relaxometric properties in response to light stimulation was performed with spiropyran-DO3A-Gd in water solution after brief light pulses.



\subsection{Illumination with Two Different LED's}

Considering the differences in the peak absorption bands of non-complexed and complexed spiropyran-DO3A in different solvents, illumination with LEDs possessing the maximal emission at either $465 \mathrm{~nm}$ or $525 \mathrm{~nm}$ were performed. Although, clearly, matching the peak absorption band of spiropyran-DO3A and spiropyran-DO3A-Gd to the emission wavelength of the LED would give maximal response, we also sought to determine if the CA could respond to luciferase systems that only partially overlapped the excitation maximum.

It has been reported that spiropyran is most sensitive in the green spectrum, compared to illumination with a red LED [9]. The adjustment of both LEDs to an identical photon emission rate of $5.78 \times 10^{14}$ photons $^{-1}{ }^{-1}$ enables a comparative measurement. Rate constants were determined for complexed and non-complexed spiropyran-DO3A dissolved in water and ethanol after illumination using the two LEDs (Table 1). The data clearly shows, when comparing the contrast agents either dissolved in EtOH or water that increasing the distance between absorbance peak of the CA and the maximum emission of the light source significantly lowers the rate constants. However, the less effective blue emission is still able to switch the CA even at low photon densities. 
Table 1. Illumination with two LEDs: Spiropyran-DO3A and spiropyran-DO3A-Gd were illuminated with green LED possessing maximum emission at $\lambda=525 \mathrm{~nm}$ and the blue LED $(\lambda=465 \mathrm{~nm})$. The illumination power was equally adjusted to a total photon flux of $5.78 \times 10^{14}$ photons $\cdot \mathrm{s}^{-1}$. $*$ The column $\Delta \max [\mathrm{nm}]$ refers to the separation between the absorption peak of the $\mathrm{CA}$ in relation to the maximum emission peak of the LED.

\begin{tabular}{cccccc}
\hline $\begin{array}{c}\text { LED } \\
\text { emission } \\
\text { max }\end{array}$ & Solvent & $\begin{array}{c}\Delta \text { max } \\
(\mathbf{n m})\end{array}$ & $\begin{array}{c}\text { Spiropyran-DO3A rate } \\
\text { constant }\end{array}$ & $\begin{array}{c}\Delta \text { max } \\
(\mathbf{n m})\end{array}$ & $\begin{array}{c}\text { Spiropyran-DO3A-Gd rate } \\
\text { constant }\end{array}$ \\
\hline \multirow{2}{*}{$465 \mathrm{~nm}$} & Water & 31 & $1.55 \times 10^{-2} \pm\left(2.38 \times 10^{-3}\right) \mathrm{s}^{-1}$ & 5 & $1.59 \times 10^{-2} \pm\left(1.99 \times 10^{-3}\right) \mathrm{s}^{-1}$ \\
& EtOH & 53 & $2.29 \times 10^{-2} \pm\left(1.81 \times 10^{-3}\right) \mathrm{s}^{-1}$ & 13 & $1.89 \times 10^{-2} \pm\left(3.51 \times 10^{-4}\right) \mathrm{s}^{-1}$ \\
\multirow{2}{*}{$525 \mathrm{~nm}$} & Water & 29 & $1.29 \times 10^{-3} \pm\left(3.68 \times 10^{-3}\right) \mathrm{s}^{-1}$ & 55 & $6.27 \times 10^{-3} \pm\left(4.76 \times 10^{-4}\right) \mathrm{s}^{-1}$ \\
& EtOH & 7 & $1.94 \times 10^{-2} \pm\left(7.02 \times 10^{-4}\right) \mathrm{s}^{-1}$ & 47 & $8.31 \times 10^{-3} \pm\left(1.50 \times 10^{-3}\right) \mathrm{s}^{-1}$ \\
\hline
\end{tabular}

\subsection{Overexpression of Gaussia Princeps Luciferase}

After successful determination of photon emission rate and illumination time needed for inducing isomerization of the merocyanine to the spiropyran form, we evaluated the photon emission rate of the enzyme Gaussia princeps luciferase to validate if the application of light producing enzymes can induce an isomerization of the contrast agent. Overexpression of luciferases in eukaryotic cells also allows for high photon production. Values up to $5.2 \times 10^{4}$ photons $^{-1} \mathrm{~s}^{-1}$ for a single firefly luciferase overexpressing tumor cell have been described in previous literature [36]. After in vivo transplantation of luciferase overexpressing cells and the formation of tumors in vivo, values up to $1 \times 10^{11}$ photons $\cdot \mathrm{s}^{-1}$ could be shown using whole body imaging systems [36,37]. Recently it has been shown that Gaussia luciferase, isolated from the marine copepod Gaussia princeps emits brighter light than FLuc [30,38]. Therefore, we focused on this enzyme. N-terminal tagged Gaussia princeps luciferase was overexpressed under the $\mathrm{T} 7$ promoter using a bacterial expression system and purified with Ni-IDA affinity chromatography. After purification, SDS-polyacrylamide electrophoresis was performed, verifying a protein band at approximately $20 \mathrm{kDa}$, corresponding to the Gaussia princeps luciferase with a molecular weight of $19.9 \mathrm{kDa}$ (Figure 6A) [30]. Spectral analysis confirmed a maximum emission at $465 \mathrm{~nm}$ with a broad emission spectrum extending to $600 \mathrm{~nm}$ after supplementation of coelenterazine (Figure 7A), as reported previously [30]. The maximum emission wavelength corresponds to the maximum emission wavelength of the blue LED (Figure 8A). Using the optical imaging system IVIS-200 we recorded the emission kinetics of the bioluminescence reaction after supplementation of the substrate coelenterazine.

Thirty s after substrate addition we observed a bright light emission with a total photon flux of $2.289 \times 10^{11}$ photons $\cdot \mathrm{s}^{-1}$ (Figure 6B and Figure 7B). Light emission decreased rapidly in the following $60 \mathrm{~s}$ to $2.855 \times 10^{10}$ photons $\cdot \mathrm{s}^{-1}$. Purified GLuc exhibits flash kinetics, which decays rapidly over time in contrast to the enzyme firefly luciferase, which has glow kinetics [30]. Verhaegen et al. reported a light emission peak at $1 \mathrm{~s}$ after substrate addition followed by a rapid decline, dependent on the coelenterazine concentration [39]. 
Figure 6. Optical properties of overexpressed Gaussia princeps luciferase: Gaussia princeps luciferase was overexpressed in a bacterial expression system after induction with IPTG and purified using affinity chromatography. SDS-polyacrylamide electrophoresis after Ni-IDA purification displays an enriched protein amount (A). $1.36 \mu \mathrm{g}$ total protein amount was used for analysis. Determination of total photon flux was performed in an IVIS-100 imaging system over a time period of $510 \mathrm{~s}$ after supplementation of coelenterazine (B). Quantification of the total photon flux of the spots is shown in Figure 7.



A



B

Figure 7. Optical properties of overexpressed Gaussia princeps luciferase: Gaussia princeps luciferase was overexpressed in a bacterial expression system after induction with IPTG and purified using affinity chromatography. Analysis of the emission spectrum displays the maximum intensity at $465 \mathrm{~nm}$ (A). Determination of total photon emission rate was performed in an IVIS-100 imaging system over a time period of $510 \mathrm{~s}$ (B).
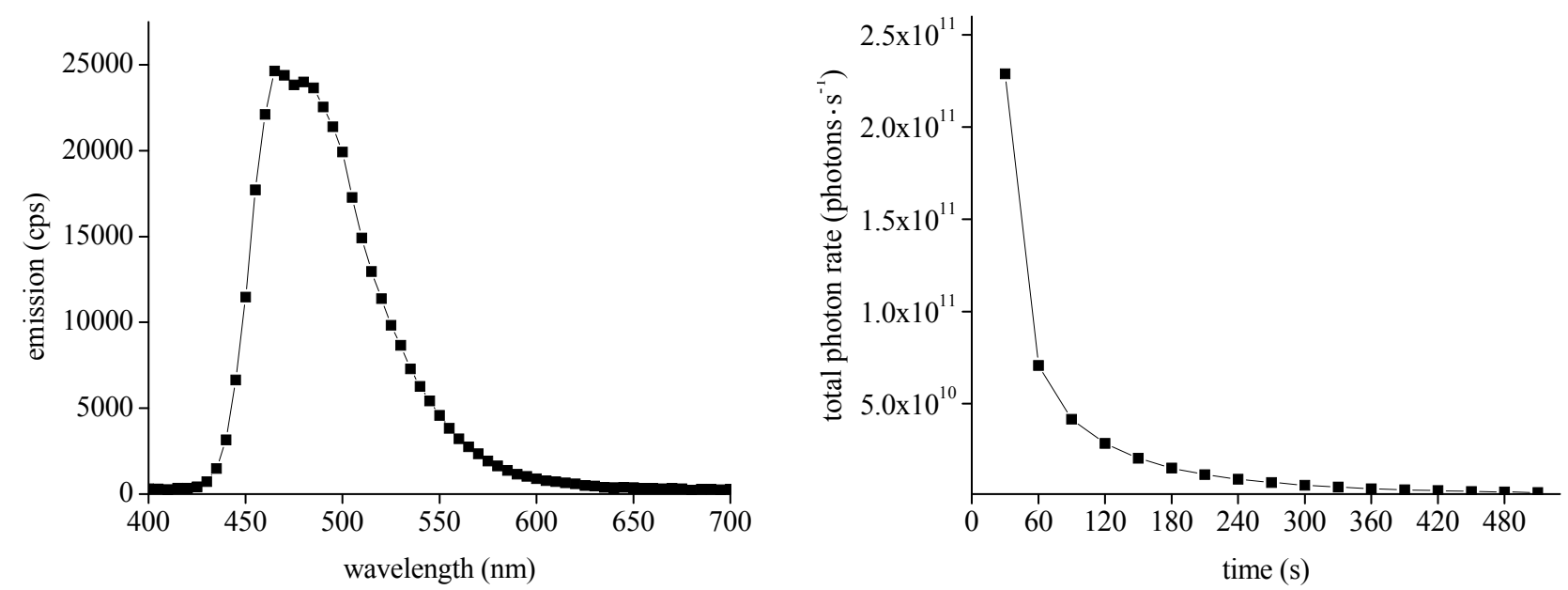

A 
Figure 8. Spectral characteristics of LEDs: The spectral characteristics of the blue-emitting LED $\lambda_{\max }=465 \mathrm{~nm}$ and the green emitting LED $\lambda_{\max }=525 \mathrm{~nm}$ were recorded at the operating current of $18.5 \mathrm{~mA}(\mathbf{A})$. Operating current versus photon emission rate is displayed in $(\mathbf{B})$.


A

$\mathrm{B}$

Producing appreciable quantities of properly folded GLuc has been reported to be difficult, primarily because of the presence of multiple disulfide bonds [40]. Goerke et al. showed that $5 \mu \mathrm{g} \cdot \mathrm{mL}^{-1}$ of GLuc produced a specific activity of $9.1 \times 10^{22}$ photons $\mathrm{s}^{-1} \mathrm{~mol}^{-1}$, corresponding to a total activity of $2.5 \times 10^{13}$ photons s ${ }^{-1} \mathrm{~mL}^{-1}$, using a bacterial expression system and a prokaryotic signal peptide for periplasmatic expression [40]. Welsh et al. reported the effective application of cell-free protein synthesis (CFPS) for the production of GLuc, possessing a specific activity of $9.0 \times 10^{23}$ photons s $^{-1} \mathrm{~mol}^{-1}$ and a half life of $1.3( \pm 0.3) \min$ [38]. The short half-life of the Gaussia luciferase enzyme leads to the question whether it is enough to influence the MC to SP interconversion. Recently, GLuc mutants with a considerably prolonged half life of $13.5( \pm 4)$ min have been produced that possess a high specific activity of $8.1 \times 10^{23}$ photons s${ }^{-1} \mathrm{~mol}^{-1}$ [38]. Indeed, these values indicate that the amount of light produced by luciferase is in the range of the minimal total photon emission rate necessary to induce a conformational change of spiropyran-DO3A-Gd.

In principle, two different possible mechanisms of excitation of a photosensitive molecule exist: Radiative absorption of the bioluminescence emission and direct energy transfer to the photosensitive molecule [41]. Theodossiou et al. and Carpenter et al. have shown that the overexpression of FLuc is capable to activate photosensitive molecules used as photosensitizers [41,42]. In preliminary experiments we could not observe a direct energy transfer of the Foerster type or radiative absorption after mixing coelenterazine, GLuc and spiropyran-DO3A-Gd (data not shown). Also an external illumination using various enzyme concentrations could not induce a MC to SP isomerization (data not shown). But improvements of the enzyme production and prolonging the half-life of GLuc could clearly lead to a MC to SP isomerization. We are currently working on improvements to the DO3A-tethered photochromic molecules to improve light sensitivity as well as mutations to the luciferases to improve light production and prolonging enzymatic half life. 


\section{Experimental}

Synthesis of spiropyran conjugated to the macrocyclic ligand 1,4,7,10-tetraazacyclododecane 1,4,7-triacetic acid (spiropyran-DO3A) was performed as previously reported [8]. For Gd(III) complexation, a mixture of spiropyran-DO3A $(0.124 \mathrm{~g})$ and $\mathrm{Gd}(\mathrm{OTf})_{3}(0.11 \mathrm{~g})$ in methanol $(6 \mathrm{~mL})$ was stirred for $24 \mathrm{~h}$ at $60{ }^{\circ} \mathrm{C}$. The solvent was evaporated in vacuo. The orange-colored solid powder was stored at room temperature in the dark for long-term storage. Prior to use, the powder was rehydrated in water (ultra-pure grade, Cellgro, Manassas, VA, USA) and stored at $4{ }^{\circ} \mathrm{C}$ for short term use. Solution was used for a maximum of 14 days after rehydration. Samples were prepared either in water or ethanol (Sigma-Aldrich, St. Louis, MO, USA).

The crowned spiropyran and crowned spiropyran complexed with Gd(III) ions were dissolved in water and further diluted in water or $100 \% \mathrm{EtOH}$ to $50.58( \pm 11.77) \mu \mathrm{M}$ for spiropyran-DO3A and $44.32( \pm 6.09) \mu \mathrm{M}$ for spiropyran-DO3A-Gd. This resulted in peak absorbance values between 0.6 to 0.5 for the aqueous solutions and 0.6 to 0.7 for the ethanol solutions.

The UV-visible spectra were recorded with an UV-Vis spectrophotometer (Cary 100Bio) in quartz cells (Starna Cells, Atascadero, CA, USA, catalog number 18/9-Q-10) in $250 \mu \mathrm{L}$ of solution. For the illumination experiments a blue LED (650 millicandela typical intensity, viewing angle of 154 degrees and maximal emission at $465 \mathrm{~nm}$, RadioShack, Fort Worth, Texas, USA catalog number 276-013) and a green LED (960 millilumen typical intensity, viewing angle of 130 degrees and maximal emission at $525 \mathrm{~nm}$, RadioShack catalog number 276-027) were used. The illuminance by the LED was adjusted with the constant current source. The current source was a $9 \mathrm{~V}$ regulated voltage source (TENMA, Newark, Palatine, IL, USA) and calibrated resistors placed in series with the LED to produce currents up to $18.5 \mathrm{~mA}$ (measured by Tektronix TX3 multimeter; Figure 9). The largest current produces no heating induced effects in the emission spectra. The spectral characteristics of the blue-emitting LED and the green emitting LED are displayed in Figure 8A. The relationship between the operating current and the photon rate is shown in Figure 8B.

The LED has a rectangular package. The LED package was positioned next to the surface of the quartz cell, ensuring a maximum light illumination to the interior, fluid containing gap of the quartz cell. Irradiation was performed for defined time periods ( $1 \mathrm{~s}$ up to $10 \mathrm{~min}$ ). The cuvette was briefly removed from the holder, placed in the UV-Vis spectrophotometer followed by UV-visible spectroscopic measurement. The emission spectra (between $400 \mathrm{~nm}$ and $650 \mathrm{~nm}$ ) produced during the illumination period were recorded. For control conditions, quartz cells subjected to the same number of measurements without LED light illumination were performed. The kinetic rate constants for the lightinduced isomerization from the merocyanine to the spiropyran form were extrapolated from the slope of the plot as described [43]:

$$
-\mathrm{kt}=\ln \frac{\mathrm{A}_{\mathrm{t}}}{\mathrm{A}_{0}}
$$

where $A_{t}=$ peak absorption value at the time point $t$ after illumination and $A_{0}=$ peak absorption value before illumination The total LED power emitted was determined with a calibrated optical power meter (Newport Corp840 and sensor 818-SC). The LED was placed with minimal distance in front of the sensor to simulate comparable illumination conditions as used for the quartz cell illumination. 
Calibration of the power meter was performed at the maximum emission wavelengths of the LEDs (blue: $465 \mathrm{~nm}$, green: $525 \mathrm{~nm}$ ). The LED emission spectra was measured with an optical spectrum analyzer (Ando, now Yokogawa, AQ-6315A, Sugar Land, TX, USA) and then scaled to the total LED emission power to produce power distribution as a function of wavelength $\mathrm{W}_{\lambda}$.

Figure 9. Determination of current: Different resistors between $330 \Omega$ and $220 \mathrm{k} \Omega$ were connected with the LED in series. For experiments a $9 \mathrm{~V}$ voltage supply was used. The voltage drop at the resistor was measured and the resulting current calculated.



The photon emission rate was measured in the following manner: The photon emission rate per nanometer at a fixed current is:

$$
\text { photon rate } \mathrm{s}^{-1} \mathrm{~nm}^{-1}=\frac{W_{\lambda}}{\frac{h c}{\lambda}}
$$

and the total photon rate emitted by the LED over the emission spectrum is:

$$
\text { total photon rate } s^{-1}=\int_{\lambda_{1}}^{\lambda_{2}} \frac{W_{\lambda}}{\lambda} d \lambda
$$

where $\mathrm{h}=6.63 \times 10^{-34} \mathrm{~J} \cdot \mathrm{s}$ (Planck's constant, $\mathrm{c}=3.00 \times 10^{8} \mathrm{~m} \cdot \mathrm{s}^{-1}$ (velocity of light) and $\lambda$ is in $\mathrm{nm}$. Total amount of photons were determined using the integral of (2) from $\lambda_{1}=450 \mathrm{~nm}$ to $\lambda_{2}=650 \mathrm{~nm}$ (green LED) and from $\lambda_{1}=400 \mathrm{~nm}$ to $\lambda_{2}=554 \mathrm{~nm}$ (blue LED) (3).

The relationship between the diode current, electrical power and total photon emission rate for the calibrated resistors used in the current investigation is displayed in Table 2 for the blue LED and for the green LED: 
Table 2. Illumination with two LEDs: Diode current, electrical power and total photon emission rate were determined for the blue emitting LED and the green emitting LED.

\begin{tabular}{ccccc}
\hline & \multicolumn{2}{c}{ Blue emitting LED } & \multicolumn{2}{c}{ Green emitting LED } \\
\hline $\begin{array}{c}\text { Current } \\
(\mathrm{mA})\end{array}$ & $\begin{array}{c}\text { Total radiant } \\
\text { flux }(\mu \mathrm{W})\end{array}$ & $\begin{array}{c}\text { Photon emission rate } \\
\left.\left(\text { photons }^{-1}\right)^{-1}\right)\end{array}$ & $\begin{array}{c}\text { Total radiant } \\
\text { flux }(\mu \mathrm{W})\end{array}$ & $\begin{array}{c}\text { Photon emission rate } \\
\left(\text { photons } \cdot \mathrm{s}^{-1}\right)\end{array}$ \\
\hline 17.676 & 7536 & $1.7542 \times 10^{16}$ & 2467.615 & $6.5372 \times 10^{15}$ \\
6.314 & 2927.58 & $6.8873 \times 10^{15}$ & 1133.615 & $2.9919 \times 10^{15}$ \\
1.3978 & 588.72 & $1.3702 \times 10^{15}$ & 298.405 & $7.9035 \times 10^{14}$ \\
0.6687 & 248.4 & $5.7821 \times 10^{14}$ & 151.08 & $4.0141 \times 10^{14}$ \\
0.3031 & 82.884 & $1.9289 \times 10^{14}$ & 66.3875 & $1.7577 \times 10^{14}$ \\
0.1443 & 26.334 & $6.1284 \times 10^{13}$ & 29.29 & $7.7539 \times 10^{13}$ \\
0.0984 & 13.46 & $3.132 \times 10^{13}$ & 18.93 & $5.0114 \times 10^{13}$ \\
0.0675 & 6.4596 & $1.5027 \times 10^{13}$ & 12.15 & $3.2162 \times 10^{13}$ \\
0.0309 & 1.0182 & $2.37 \times 10^{13}$ & 4.634 & $1.2267 \times 10^{13}$ \\
\hline
\end{tabular}

$T_{1}$ measurements were performed at constant temperature of $37{ }^{\circ} \mathrm{C}$ with a relaxometer (Bruker minispec, mq60, Bruker Biospin, Rheinstetten, Germany). Adjustments for measuring $T_{1}$ values for the determination of $r_{1}$ were: First pulse separation $=30 \mathrm{~ms}$; final pulse separation $=13,000 \mathrm{~ms}$; number of data points used for fitting $=12$; delay sampling window $=0.03 \mathrm{~ms}$; sampling window $=0.015 \mathrm{~ms}$; time for saturation curve display $=3 \mathrm{~s}$; total analysis time $=12.16 \mathrm{~min}$. Concentrations of spiropyran-DO3A-Gd between $39.4 \mu \mathrm{M}$ and $157.6 \mu \mathrm{M}$ were used in a total of $400 \mu \mathrm{L}$ water in flat-bottom NMR-tubes with a diameter of $0.6 \mathrm{~mm}$. The $r_{1}$ values were determined after plotting $1 / T_{1}$ values against the respective varying concentrations of the contrast agent, either kept in the dark or illuminated. Before the measurement, samples were adjusted to $37{ }^{\circ} \mathrm{C}$. For the determination of $\Delta \mathrm{T}_{1}$ values after illuminating with different photon emission rates and different time periods, a different measurement-sequence was used to reduce the analysis time from $12.16 \mathrm{~min}$ to $7.15 \mathrm{~min}$. Additional parameters were: First pulse separation $=10 \mathrm{~ms}$; final pulse separation $=10,000 \mathrm{~ms}$; number of data points used for fitting $=12$; delay sampling window $=0.031 \mathrm{~ms}$; sampling window $=0.015 \mathrm{~ms}$; time for saturation curve display $=3 \mathrm{~s}$.

The 591 bp cDNA of humanized Gaussia princeps luciferase (NanoLight, Pinetop, AZ, USA) was amplified with Polymerase Chain Reaction (PCR) using primers (5'-ACTGATCCATGGGAGTCAA AGTTCTGTTTGC-3' and 5'-ACAGTACTCGAGAGCGGCCGCTTGGTCACCACC-3'). Through the introduction of a 5' SalI and 3'NheI restriction site, the gene was introduced in frame into the expression vector pET28b (Addgene, Cambridge, MA, USA), now bearing a 3'-terminal polyhistidine tag (5'-CAGCAGCAGCAGCAGCAG-3'). Subcloning was performed in NEB5-alpha bacterial strain and verification was performed with restriction analysis using SacII und MluI and DNA sequencing. As protein expression system, competent JM109 (DE3) cells were transformed with the plasmid. Overexpression was started at a $\mathrm{OD}_{600} \mathrm{~nm}$ of 0.4 after induction with $1.0 \mathrm{M}$ isopropyl $\beta$-D-1-thiogalactopyranoside (IPTG) and continuously stirred at $15{ }^{\circ} \mathrm{C}$ overnight. The bacterial culture was harvested at an $\mathrm{OD}_{600 \mathrm{~nm}}$ of 0.8 and centrifuged in a Sorvall RC-5BPlus centrifuge (Thermo Scientific, Asheville, NC, USA, USA) equipped with a GSA rotor at 2,700 $\times g$. Bacterial pellets were resuspended in Tris-buffered saline, $\mathrm{pH}=7.6$, sonicated and purified with a Protino Ni-IDA column (Macherey-Nagel, Bethlehem, PA, USA). Protein concentration was determined with the BCA assay 
(Pierce Biotechnology, Rockford, IL, USA) in a microplate reader (TecanSafire ${ }^{2}$, Tecan Group Ltd., Männedorf, Switzerland) at $562 \mathrm{~nm}$. Validation of the product was performed with SDS-polyacrylamide electrophoresis (Bio-Rad, Hercules, CA, USA) and a 7-175 kDa marker (catalog-number P7709V, New England Biolabs, Ipswich, MA, USA) as reference. Emission spectra were recorded with the HORIBA Jobin Yvon Fluoro Max-P spectrophotometer after supplementation of coelenterazine (Wako Chemicals USA, Inc, Richmond, VA, USA). Coelenterazine stock solution was prepared in EtOH and further diluted in TBS buffer. Optical imaging was performed in an IVIS-100 system (Caliper Life Science, Hopkinton, MA, USA). Parameters used for measurements: Open filter, field of view 10, f-stop 16, exposure time $5 \mathrm{~s}$ and binning 4. Repetitive measurements were performed after adding the substrate with a time delay of approximately $25 \mathrm{~s}$ between each measurement. Protein lysates were placed into a white colored 96 multiwell polysterene plate (Nalge Nunc International, Rochester, NY, USA) prior measurement. We calculated the total photon rate (equivalent to the total flux) based on a region of interest (ROI) measurement. The radiance in each pixel was integrated over the ROI and multiplied with $4 \pi$. A ROI area of $0.419\left( \pm 1.89 \times 10^{-3}\right) \mathrm{cm}^{-2}$ was used.

\section{Conclusions}

The work performed here describes, as a longterm goal, a method to noninvasively map gene expression in deep tissues in vivo by developing magnetic resonance imaging contrast agents (MRI CAs) that are responsive to commonly employed luminescent biomarker systems. The results reported here show promise that a reversible MRI CA that responds to luciferase is feasible. Moreover, using LEDs to mimic bioluminescence emission has proven to be a very effective model system to experimentally induce photochromic molecule isomerization and comparison with a biological system. Application of the spiropyran-DO3A-Gd CA will be of great utility to allow for deep tissue probing of gene expression using non-invasive imaging methods.

\section{Acknowledgments}

The authors would like to acknowledge funding from the National Institutes of Health (EB000993). The research was also supported in part by the National Science Foundation (ECS 0823827).

\section{Conflict of Interest}

The authors declare no conflict of interest.

\section{References and Notes}

1. Lukyanov, B.S.; Lukyanova, M.B. Spiropyrans: Synthesis, Properties, and Application. (Review). Chem. Heterocyc. Compd. 2005, 41, 281-311.

2. Tu, C.Q.; Osborne, E.A.; Louie, A.Y. Synthesis and characterization of a redox- and light-sensitive MRI contrast agent. Tetrahedron 2009, 65, 1241-1246.

3. Garcia, A.A.; Cherian, S.; Park, J.; Gust, D.; Jahnke, F.; Rosario, R. Photon-controlled phase partitioning of spiropyrans. J. Phys. Chem. A 2000, 104, 6103-6107. 
4. Sumaru, K.; Satoh, T.; Takagi, T.; Takai, K.; Kanamori, T. Isomerization of spirobenzopyrans bearing electron-donating and electron-withdrawing groups in acidic aqueous solutions. Phys. Chem. Chem. Phys. 2011, 13, 7322-7329.

5. Sunamoto, J.; Iwamoto, K.; Akutagawa, M.; Nagase, M.; Kondo, H. Rate Control by Restricting Mobility of Substrate in Specific Reaction Field-Negative Photochromism of Water-Soluble Spiropyran in Aot Reversed Micelles. J. Am. Chem. Soc. 1982, 104, 4904-4907.

6. Salhin, A.M.A.; Tanaka, M.; Kamada, K.; Ando, H.; Ikeda, T.; Shibutani, Y.; Yajima, S.; Nakamura, M.; Kimura, K. Decisive factors in the photoisomerization behavior of crowned spirobenzopyrans: Metal ion interaction with crown ether and phenolate anion moieties. Eur. J. Org. Chem. 2002, 655-662.

7. Fedorova, O.A.; Gromov, S.P.; Alfimov, M.V. Cation-dependent pericyclic reactions of crown-containing photochromic compounds. Russ. Chem. Bull. 2001, 50, 1970-1983.

8. Tu, C.Q.; Louie, A.Y. Photochromically-controlled, reversibly-activated MRI and optical contrast agent. Chem. Commun. 2007, 1331-1333.

9. Stitzel, S.; Byrne, R.; Diamond, D. LED switching of spiropyran-doped polymer films. J. Mater. Sci. 2006, 41, 5841-5844.

10. Schaudel, B.; Guermeur, C.; Sanchez, C.; Nakatani, K.; Delaire, J.A. Spirooxazine- and spiropyran-doped hybrid organic-inorganic matrices with very fast photochromic responses. J. Mater. Chem. 1997, 7, 61-65.

11. Whelan, J.; Abdallah, D.; Wojtyk, J.; Buncel, E. Micro-environmental fine-tuning of electronic and kinetic properties of photochromic dyes. J. Mater. Chem. 2010, 20, 5727-5735.

12. Zhu, M.Q.; Zhu, L.Y.; Han, J.J.; Wu, W.W.; Hurst, J.K.; Li, A.D.Q. Spiropyran-based photochromic polymer nanoparticles with optically switchable luminescence. J. Am. Chem. Soc. 2006, 128, 4303-4309.

13. Li, Y.Y.; Fan, M.G.; Zhang, S.X.; Yao, J.N. Photochromism induced aggregate-monomer interconversion and fluorescence switch of porphyrin with spiropyran. J. Phys. Org. Chem. 2007, 20, 884-887.

14. Giordano, L.; Macareno, J.; Song, L.; Jovin, T.M.; Irie, M.; Jares-Erijman, E.A. Fluorescence resonance energy transfer using spiropyran and diarylethene photochromic acceptors. Molecules 2000, 5, 591-593.

15. Tanaka, M.; Nakamura, M.; Salhin, M.A.A.; Ikeda, T.; Kamada, K.; Ando, H.; Shibutani, Y.; Kimura, K. Synthesis and photochromism of spirobenzopyran derivatives bearing an oxymethylcrown ether moiety: Metal ion-induced switching between positive and negative photochromisms. J. Org. Chem. 2001, 66, 1533-1537.

16. Natali, M.; Aakeroy, C.; Desper, J.; Giordani, S. The role of metal ions and counterions in the switching behavior of a carboxylic acid functionalized spiropyran. Dalton T. 2010, 39, 8269-8277.

17. Scarmagnani, S.; Walsh, Z.; Slater, C.; Alhashimy, N.; Paull, B.; Macka, M.; Diamond, D. Polystyrene bead-based system for optical sensing using spiropyran photoswitches. J. Mater. Chem. 2008, 18, 5063-5071. 
18. Yoshida, J.; Watanuki, A.; Takano, H.; Kobayashi, H.; Ikeda, H.; Ogata, N. Optically-Controlled Photonic Switches Based on Spiropyran-Doped Marine-Biopolymer DNA-Lipid Complex Films. In Organic Photonic Materials and Devices VIII; Grote, J.G., Kajzar, F., Kim, N., Eds.; Proceedings of SPIE: San Jose, CA, USA, 2006.

19. Bhawalkar, J.D.; Kumar, N.D.; Zhao, C.F.; Prasad, P.N. Two-photon photodynamic therapy. J. Clin. Laser Med. Surg. 1997, 15, 201-204.

20. Wang, R.K.K.; Xu, X.Q.; Tuchin, V.V.; Elder, J.B. Concurrent enhancement of imaging depth and contrast for optical coherence tomography by hyperosmotic agents. J. Opt. Soc. Am. B 2001, 18, 948-953.

21. Ipe, B.I.; Mahima, S.; Thomas, K.G. Light-induced modulation of self-assembly on spiropyrancapped gold nanoparticles: A potential system for the controlled release of amino acid derivatives. J. Am. Chem. Soc. 2003, 125, 7174-7175.

22. Zhu, M.Q.; Zhang, G.F.; Li, C.; Aldred, M.P.; Chang, E.; Drezek, R.A.; Li, A.D. Reversible Two-Photon Photoswitching and Two-Photon Imaging of Immunofunctionalized Nanoparticles Targeted to Cancer Cells. J. Am. Chem. Soc. 2011, 133, 365-372.

23. Wilson, T.; Hastings, J.W. Bioluminescence. Annu. Rev. Cell. Dev. Biol. 1998, 14, 197-230.

24. Sadikot, R.T.; Blackwell, T.S. Bioluminescence imaging. Proc. Am. Thorac. Soc. 2005, 2, 511-512, 537-540.

25. Contag, C.H.; Jenkins, D.; Contag, P.R.; Negrin, R.S. Use of reporter genes for optical measurements of neoplastic disease in vivo. Neoplasia 2000, 2, 41-52.

26. Niwa, K.; Ichino, Y.; Kumata, S.; Nakajima, Y.; Hiraishi, Y.; Kato, D.; Viviani, V.R.; Ohmiya, Y. Quantum Yields and Kinetics of the Firefly Bioluminescence Reaction of Beetle Luciferases. Photochem. Photobiol. 2010, 86, 1046-1049.

27. Nakajima, Y.; Yamazaki, T.; Nishii, S.; Noguchi, T.; Hoshino, H.; Niwa, K.; Viviani, V.R.; Ohmiya, Y. Enhanced Beetle Luciferase for High-Resolution Bioluminescence Imaging. PLoS One 2010, 5, e10011.

28. Verhaegen, M.; Christopoulos, T.K. Recombinant Gaussia luciferase. Overexpression, purification, and analytical application of a bioluminescent reporter for DNA hybridization. Anal. Chem. 2002, 74, 4378-4385.

29. Venisnik, K.M.; Olafsen, T.; Gambhir, S.S.; Wu, A.M. Fusion of Gaussia luciferase to an engineered anti-carcinoembryonic antigen (CEA) antibody for in vivo optical imaging. Mol. Imaging. Biol. 2007, 9, 267-277.

30. Tannous, B.A.; Kim, D.E.; Fernandez, J.L.; Weissleder, R.; Breakefield, X.O. Codon-optimized Gaussia luciferase cDNA for mammalian gene expression in culture and in vivo. Mol. Ther. 2005, 11, 435-443.

31. Zhang, S.G.; Zhang, Q.H.; Ye, B.X.; Li, X.L.; Zhang, X.P.; Deng, Y.Q. Photochromism of Spiropyran in Ionic Liquids: Enhanced Fluorescence and Delayed Thermal Reversion. J. Phys. Chem. B 2009, 113, 6012-6019.

32. Pimienta, V.; Lavabre, D.; Levy, G.; Samat, A.; Guglielmetti, R.; Micheau, J.C. Kinetic analysis of photochromic systems under continuous irradiation. Application to spiropyrans. J. Phys. Chem. 1996, 100, 4485-4490. 
33. Gorner, H. Photochromism of nitrospiropyrans: Effects of structure, solvent and temperature. Phys. Chem. Chem. Phys. 2001, 3, 416-423.

34. Diamond, D.; Radu, A.; Byrne, R.; Alhashimy, N.; Fusaro, M.; Scarmagnani, S. Spiropyran-based reversible, light-modulated sensing with reduced photofatigue. J. Photochem. Photobiol. A 2009, 206, 109-115.

35. Giordani, S.; Movia, D.; Prina-Mello, A.; Volkov, Y. Determination of Spiropyran Cytotoxicity by High Content Screening and Analysis for Safe Application in Bionanosensing. Chem. Res. Toxicol. 2010, 23, 1459-1466.

36. Kim, J.B.; Urban, K.; Cochran, E.; Lee, S.; Ang, A.; Rice, B.; Bata, A.; Campbell, K.; Coffee, R.; Gorodinsky, A.; et al. Non-invasive detection of a small number of bioluminescent cancer cells in vivo. PLoS One 2010, 5, e9364.

37. Yanagihara, K.; Takigahira, M.; Takeshita, F.; Komatsu, T.; Nishio, K.; Hasegawa, F.; Ochiya, T. A photon counting technique for quantitatively evaluating progression of peritoneal tumor dissemination. Cancer Res. 2006, 66, 7532-7539.

38. Welsh, J.P.; Patel, K.G.; Manthiram, K.; Swartz, J.R. Multiply mutated Gaussia luciferases provide prolonged and intense bioluminescence. Biochem. Biophys. Res. Commun. 2009, 389, 563-568.

39. Verhaegent, M.; Christopoulos, T.K. Recombinant Gaussia luciferase. Overexpression, purification, and analytical application of a bioluminescent reporter for DNA hybridization. Anal. Chem. 2002, 74, 4378-4385.

40. Goerke, A.R.; Loening, A.M.; Gambhir, S.S.; Swartz, J.R. Cell-free metabolic engineering promotes high-level production of bioactive Gaussia princeps luciferase. Metab. Eng. 2008, 10, 187-200.

41. Theodossiou, T.; Hothersall, J.S.; Woods, E.A.; Okkenhaug, K.; Jacobson, J.; MacRobert, A.J. Firefly luciferin-activated rose bengal: In vitro photodynamic therapy by intracellular chemiluminescence in transgenic NIH 3 T3 cells. Cancer Res. 2003, 63, 1818-1821.

42. Carpenter, S.; Fehr, M.J.; Kraus, G.A.; Petrich, J.W. Chemiluminescent activation of the antiviral activity of hypericin: a molecular flashlight. Proc. Natl. Acad. Sci. USA 1994, 91, 12273-12277.

43. Yao, J.; Liu, Y.; Fan, M.; Zhang, S.; Sheng, X. Basic amino acid induced isomerization of a spiropyran: Towards visual recognition of basic amino acids in water. New J. Chem. 2007, 31, $1878-1881$.

Sample Availability: Samples of the compounds spiropyran-DO3A and spiropyran-DO3A-Gd are available from the authors.

(C) 2012 by the authors; licensee MDPI, Basel, Switzerland. This article is an open access article distributed under the terms and conditions of the Creative Commons Attribution license (http://creativecommons.org/licenses/by/3.0/). 